\title{
Pointwise Encoding Time Reduction with Radial Acquisition with Subtraction-Based MRA during the Follow-Up of Stent- Assisted Coil Embolization of Anterior Circulation Aneurysms
}

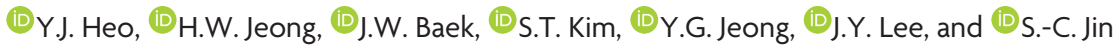

\begin{abstract}
BACKGROUND AND PURPOSE: Time-of-flight MR angiography, though widely used after coil embolization, is associated with limitations owing to magnetic susceptibility and radiofrequency shielding following stent-assisted coil embolization. We evaluated the pointwise encoding time reduction with radial acquisition (PETRA) sequence in subtraction-based MRA (qMRA) using an ultrashort TE relative to TOF-MRA during the follow-up of stent-assisted coil embolization for anterior circulation aneurysms.
\end{abstract}

MATERIALS AND METHODS: Twenty-five patients ( 3 men and 22 women; mean age, $59.1 \pm 14.0$ years) underwent stent-assisted coil embolization for anterior circulation aneurysms and were retrospectively evaluated using TOF-MRA and PETRA qMRA data from the same follow-up session. Two neuroradiologists independently reviewed both MRA findings and subjectively graded flow within the stents (relative to the latest DSA findings) and occlusion status (complete occlusion or neck/aneurysm remnant). Interobserver and intermodality agreement for TOF-MRA and PETRA qMRA were evaluated.

RESULTS: The mean score for flow visualization within the stents was significantly higher in PETRA qMRA than in TOF-MRA ( $P<.001$ for both observers), and good interobserver agreement was reported $(\kappa=0.63)$. The aneurysm occlusion status of PETRA qMRA (observer 1 , 92.0\%; observer 2, 88.0\%) was more consistent with DSA than with TOF-MRA (observer 1,76.0\%; observer 2, 80.0\%), and there was a better intermodality agreement between DSA and PETRA qMRA than between DSA and TOF-MRA.

CONCLUSIONS: These findings indicate that PETRA GMRA is a useful follow-up technique for patients who have undergone stentassisted coil embolization for anterior circulation aneurysms.

ABBREVIATIONS: PETRA = pointwise encoding time reduction with radial acquisition; qMRA = subtraction-based MRA

$\boldsymbol{S}_{\mathrm{in}}^{\mathrm{ten}}$ tent-assisted coil embolization has been widely used for cases in which conventional coil embolization is considered difficult, such as in wide-neck aneurysms. Considering the possibility of recanalization, follow-up imaging is important even after complete occlusion has been achieved. DSA is considered the criterion standard for follow-up to evaluate intra-aneurysmal flow or instent restenosis; however, this technique has risks associated with contrast media, procedural invasiveness, and radiation exposure. Contrast-enhanced MRA provides visualization of the remnant neck and stented segments that is similar to that of DSA. ${ }^{1-3}$ How-

Received November 23, 2018; accepted after revision March 11, 2019.

From the Departments of Radiology (Y.J.H., H.W.J., J.W.B.), Neurosurgery (S.T.K., Y.G.J.), and Internal Medicine (J.Y.L.), Inje University Busan Paik Hospital, Busan, Korea; and Department of Neurosurgery (S.-C.J.), Inje University Haeundae Paik Hospital, Busan, Korea.

This work was supported by the 2018 Inje University research grant.

Please address correspondence to Hae Woong Jeong, MD, PhD, Department of Radiology, Inje University Busan Paik Hospital, Bokji-ro 75, Busanjin-Gu, Busan, Korea, 47392; e-mail: hwjeong2000@hanmail.net

http://dx.doi.org/10.3174/ajnr.A6035 ever, contrast-enhanced MRA has contrast-associated risks, including nephrogenic systemic fibrosis, anaphylaxis, gadolinium deposition, and the potential for misdiagnosing perianeurysmal enhancement as recanalization.

Nonenhanced TOF-MRA is another established technique used for follow-up after coil embolization. Specifically, it is useful for the evaluation of the residual lumen of aneurysms. ${ }^{4}$ However, this approach is difficult in cases with stent-assisted coil embolization because visualizing blood flow within intracranial stents and the parent artery is complicated by radiofrequency shielding and susceptibility artifacts. ${ }^{2,5}$ These can obscure the neck remnant and adjacent structures as a result of signal loss. Several trials have attempted to overcome these problems when using MRA after stent-assisted coil embolization. ${ }^{6-10}$ For example, ultrashort-TE MRA is known to decrease the susceptibility artifacts caused by metallic devices and to increase the signal intensity within the stent. ${ }^{11,12}$ Recently, a few studies ${ }^{7-9}$ have highlighted the efficacy of Silent MRA (GE Healthcare, Milwaukee, Wisconsin) using an ultrashort TE after stent-assisted coil embolization. 
The present study evaluated another nonenhanced MRA technique using ultrashort TE, known as pointwise encoding time reduction with radial acquisition (PETRA). Our results indicate that PETRA showed a higher SNR compared with the conventional ultrashort TE. This was due to a shorter encoding time for whole $k$-space, which was required before the signal decayed. ${ }^{13}$ To the best of our knowledge, PETRA with subtraction-based MRA (qMRA) has not been studied following stent-assisted coil embolization. Therefore, we aimed to evaluate its utility relative to TOF-MRA during follow-up after stent-assisted coil embolization for anterior circulation aneurysms.

\section{MATERIALS AND METHODS Patients}

This retrospective study evaluated 25 patients ( 22 women and 3 men; mean age, $59.1 \pm 14.0$ years) who underwent stent-assisted coil embolization for unruptured aneurysms in the anterior circulation ( 24 cases in the paraclinoid segment of the ICA, 1 case in the anterior communicating artery) between September 2017 and October 2018. All subjects underwent TOF-MRA and PETRA qMRA during the same follow-up session. The retrospective protocol of the study was approved by the Inje University Busan Paik Hospital institutional review board.

Eighteen patients were treated using a Neuroform stent (Stryker Neurovascular, Kalamazoo, Michigan), 6 patients were treated with an Enterprise stent (Codman \& Shurtleff, Raynham, Massachusetts), and 1 patient was treated using both stents. Endovascular therapy and DSA were performed using a biplane angiographic unit (Integris V, Philips Healthcare, Best, the Netherlands; or Artis zee biplane system, Siemens, Erlangen, Germany). Complete occlusion was identified in 15 cases, and a residual neck was observed in 10 cases, based on the latest DSA findings.

\section{Imaging Acquisition}

Intracranial TOF-MRA and PETRA qMRA were performed during the same follow-up session using 3T MR imaging (Magnetom Skyra; Siemens) with a 64-channel head-neck coil. The PETRA qMRA technique has been described previously ${ }^{14}$ and is commercially available. Briefly, labeled and control data were obtained; the labeled data were applied to the slice-selective saturation pulse for the carotid artery (ie, arterial inflow appears dark). The saturation pulse of control data was applied to the $10 \mathrm{~mm}$ above the vertex of the head, and the subtracted MRA showed dark background tissue and suppressed venous flow. The detailed scan parameters for PETRA qMRA: TR/TE, 3.52/0.07 ms; flip angle, $6^{\circ}$; FOV, $230 \times 230 \mathrm{~mm}$; matrix, $320 \times 320$; radial sampling, 20,000 radial spokes; voxel size, $0.72 \times 0.72 \times 0.72 \mathrm{~mm}^{3}$; section thickness, $0.72 \mathrm{~mm}$; NEX, 1; bandwidth, $363 \mathrm{~Hz} /$ pixel; slice-selective saturation pulse applied once per 20 TRs; and acquisition time, 2 minutes 10 seconds. The detailed scan parameters for TOF-MRA: TR/TE, 24/3.99 ms; flip angle, $20^{\circ}$; FOV, $220 \times 177 \mathrm{~mm}$; matrix, $576 \times 302$; voxel size, $0.38 \times 0.38 \times 0.38 \mathrm{~mm}^{3}$; section thickness, $0.6 \mathrm{~mm}$; NEX, 1; bandwidth, $185 \mathrm{~Hz} /$ pixel; acquisition time, 6 minutes 40 seconds; number of slab, 6 . The maximum intensity projections for both sequences were reconstructed in the same manner as the DSA images.

\section{Image Analysis}

Two neuroradiologists independently reviewed both MRA findings at 2-week intervals and subjectively graded the visualization of flow within each stent (relative to the latest DSA findings) using the following scale: 1 , no signal within the stent; 2 , slightly visible but not of diagnostic quality due to image blurring; 3 , good quality with minimal blurring; or 4, excellent quality. The occlusion status of the coiled aneurysm was also evaluated. The locations of the aneurysms were provided to the observers, but they were blinded to the DSA results. However, observers were not blinded to the type of MR images because the $2 \mathrm{MR}$ images are distinctively different in appearance. One experienced interventional neuroradiologist also assessed the occlusion status of the coiled aneurysm using the Montreal Neurological Institute scale. ${ }^{15}$ Specifically, patients were rated as having complete occlusion, subtotal occlusion (residual neck, residual aneurysm), or incomplete occlusion. The latest DSA images were used as references, and the average interval between the latest DSA and the MRA follow-up was 16 months, with a range of 1 day to 127 months.

\section{Statistical Analyses}

Continuous variables are presented as mean \pm SD. We compared the mean scores for each MRA technique and type of intracranial stent using the Wilcoxon signed rank test and the Mann-Whitney test. Weighted $\kappa$ coefficients were used to evaluate the interobserver and intermodality agreement for TOF-MRA and PETRA qMRA. The results were classified as poor $(<0.20)$, fair $(0.21-$ $0.40)$, moderate (0.41-0.60), good (0.61-0.80), or excellent (0.81-1.00). ${ }^{16}$ All statistical analyses were performed using SPSS software (Version 24.0; IBM, Armonk, New York) and MedCalc for Windows (Version 18.11.3; MedCalc Software, Mariakerke, Belgium). $P$ values $<.05$ were considered statistically significant.

\section{RESULTS}

The mean score for assessing blood flow within the intracranial stent was significantly higher in the PETRA qMRA than in the TOF-MRA (observer one: $3.40 \pm 0.87$ for PETRA qMRA and $2.20 \pm 0.87$ for TOF-MRA, $P<.001$; observer two: $3.60 \pm 0.76$ for PETRA qMRA and $2.44 \pm 0.77$ for TOF-MRA, $P<.001$ ) with good interobserver agreement for both MRA techniques $(\kappa=$ 0.63 for PETRA qMRA, 95\% CI, $0.35-0.90 ; \kappa=0.63$ for TOFMRA, 95\% CI, 0.43-0.83). The Neuroform stents (open-cell design) were associated with higher scores than the Enterprise stents (closed-cell design) for both PETRA qMRA and TOF-MRA; however, no significant differences were detected between the 2 intracranial stents (Table 1). On the basis of the latest DSA findings, complete occlusion was achieved in 15 cases $(60.0 \%)$ and a residual neck was observed in 10 cases $(40.0 \%)$. PETRA qMRA findings were consistent with DSA findings in 23 cases $(92.0 \%)$ according to observer 1 and 22 cases (88.0\%) according to observer 2. Therefore, the intermodality agreement between PETRA qMRA and DSA was excellent according to observer 1 (weighted $\kappa=0.83 ; 95 \%$ CI, $0.60-1.00)$ and good according to observer 2 (weighted $\kappa=0.73 ; 95 \%$ CI, 0.47-1.00) (Table 2). However, only 19 cases $(76.0 \%)$ of TOF-MRA findings were consistent with DSA according to observer 1 and 20 cases (80.0\%) according to ob- 
server 2 . Thus, the intermodality agreement between TOF-MRA and DSA was moderate in both observer 1 (weighted $\kappa=0.44$; 95\% CI, 0.12-0.77) and observer 2 (weighted $\kappa=0.55$; 95\% CI, 0.23-0.86) (Figs 1 and 2).

\section{DISCUSSION}

The present study revealed that PETRA qMRA provided better visualization of intracranial stent flow than TOF-MRA, regardless of stent type. Furthermore, PETRA qMRA showed higher intermodality agreement with DSA than TOF-MRA. In the 2 cases in which PETRA qMRA failed to detect the residual neck, the intervals between the last DSA and the MRA were 31 and 15 months. Santillan et $\mathrm{al}^{17}$ reported cumulative occlusion after the first treatment, and our cases may have involved progressive occlusion within the aneurysms. In cases in which PETRA qMRA identified the residual neck, its configuration was more similar to DSA find-

Table 1: Comparison of PETRA qMRA and TOF-MRA according to stent design ${ }^{\mathrm{a}}$

\begin{tabular}{lccc}
\hline & $\begin{array}{c}\text { Enterprise } \\
(\boldsymbol{n}=\mathbf{6})\end{array}$ & $\begin{array}{c}\text { Neuroform } \\
(\boldsymbol{n}=\mathbf{1 8})\end{array}$ & $\begin{array}{c}\boldsymbol{P} \\
\text { Value }\end{array}$ \\
\hline PETRA qMRA & & & \\
$\quad$ Observer 1 & $2.83 \pm 1.17$ & $3.50 \pm 0.71$ & .224 \\
Observer 2 & $3.33 \pm 0.82$ & $3.89 \pm 0.32$ & .156 \\
$\quad$ Mean for 2 observers & $3.08 \pm 0.97$ & $3.69 \pm 0.42$ & .177 \\
TOF-MRA & & & \\
$\quad$ Observer 1 & $2.00 \pm 0.90$ & $2.33 \pm 0.84$ & .454 \\
$\quad$ Observer 2 & $2.17 \pm 0.41$ & $2.61 \pm 0.78$ & .137 \\
$\quad$ Mean for 2 observers & $2.08 \pm 0.58$ & $2.47 \pm 0.78$ & .224 \\
\hline
\end{tabular}

${ }^{\mathrm{a}}$ Data are presented as means.

Table 2: Comparison of occlusion status of coiled aneurysms between PETRA qMRA and TOF-MRA

\begin{tabular}{lcc}
\hline & PETRA qMRA & TOF-MRA \\
\hline Observer 1 & & \\
Complete occlusion & $100.0 \%(17 / 15)$ & $100.0 \%(21 / 15)$ \\
Residual neck & $80.0 \%(8 / 10)$ & $40.0 \%(4 / 10)$ \\
Observer 2 & & \\
Complete occlusion & $100 \%(17 / 15)$ & $100.0 \%(20 / 15)$ \\
Residual neck & $70.0 \%(7 / 10)$ & $50.0 \%(5 / 10)$ \\
Interobserver agreement & $0.90(95 \% \mathrm{Cl}$, & $0.86(95 \% \mathrm{Cl}$, \\
& $0.72-1.00)$ & $0.61-1.00)$ \\
\hline
\end{tabular}

${ }^{a}$ Data represent sensitivity (number of cases).
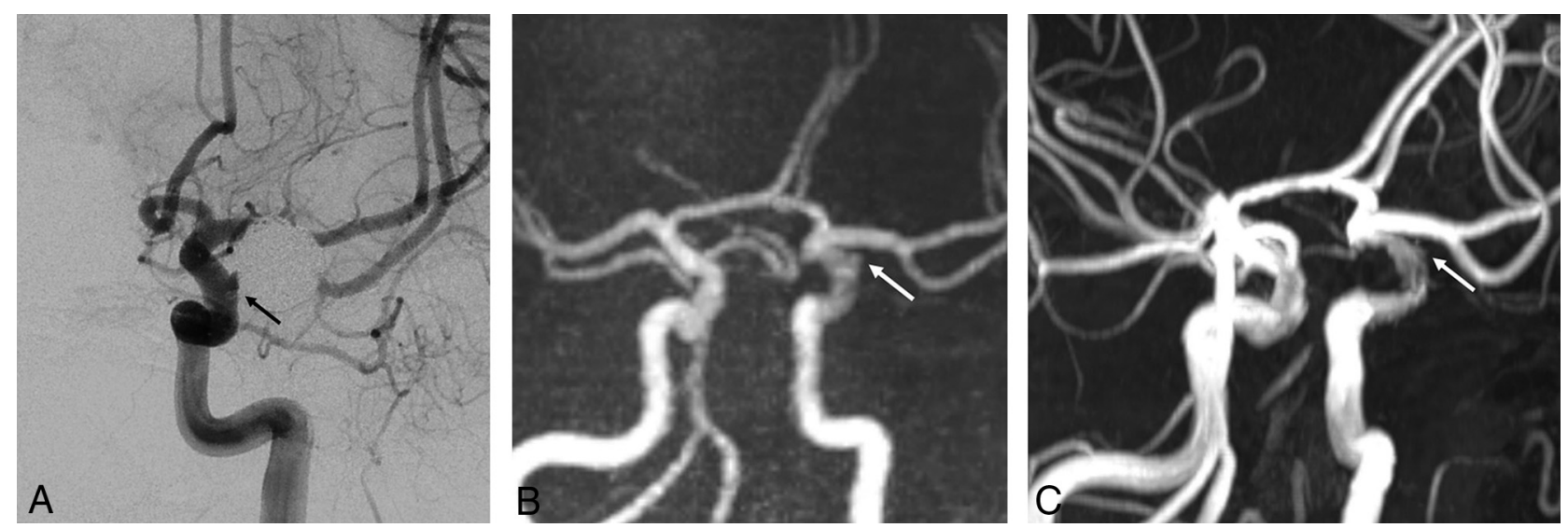

FIG 1. A 54-year-old man with a left paraclinoid internal carotid artery aneurysm who underwent stent-assisted coil embolization using a Neuroform stent. A, The follow-up DSA reveals the neck remnant (arrow). B, The PETRA qMRA reveals minimal signal loss at the stent insertion site, and the neck remnant is clearly visible (arrow). C, The TOF-MRA reveals mild signal loss at the stent insertion site and a less prominent neck remnant relative to the PETRA qMRA. 

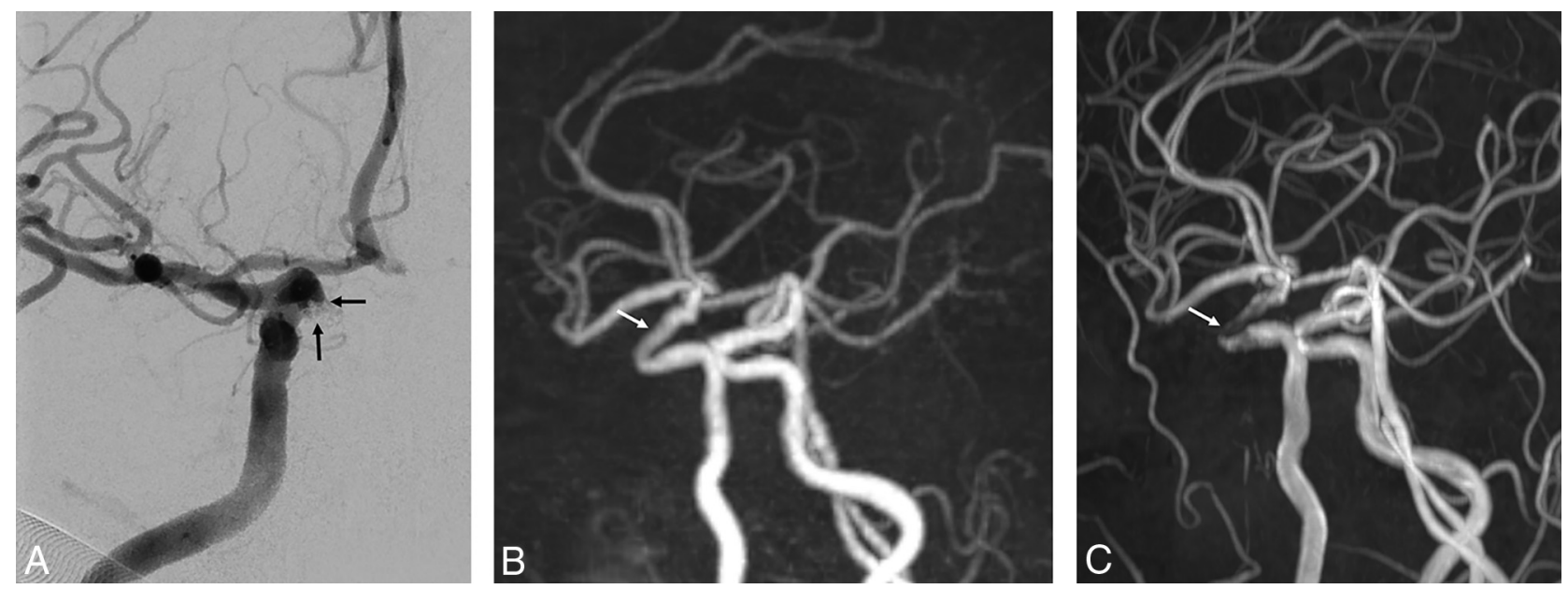

FIG 2. A 70-year-old woman with a right paraclinoid internal carotid artery aneurysm who underwent stent-assisted coil embolization using an Enterprise stent. $A$, The follow-up DSA reveals the neck remnant (arrows). $B$, The PETRA qMRA reveals minimal signal loss at the stent-insertionsite remnant (arrow), and the neck remnant is clearly visible. $C$, The TOF-MRA reveals marked signal loss at the stent-insertion-site remnant (arrow) and poor differentiation of the prominent neck remnant relative to PETRA qMRA.

imaging quality than TOF-MRA, which is another disadvantage of PETRA qMRA, like Silent MRA. Although, Silent MRA is associated with better background suppression, it has been reported that it showed more blurring and a lower SNR than TOF-MRA because of its longer readout time. ${ }^{25}$ Conversely, PETRA qMRA had a shorter acquisition time (approximately 2 minutes) than Silent MRA and TOF-MRA (approximately 6-7 minutes) using a highly undersampled outer $k$-space. ${ }^{13}$

The degree of artifacts associated with an intracranial stent can vary according to the material, cell design, and strut thickness. For example, previous reports have indicated that stents made using stainless steel or cobalt are associated with more artifacts than nitinol stents. ${ }^{10,26,27}$ The present study included only patients who were treated with nitinol stents, albeit with different cell designs (open cell versus closed cell) and strut thicknesses. Although the Neuroform stent has an open-cell design, thinner struts, and better intrastent flow than the Enterprise stent (a closed-cell design with thicker struts), there were no significant differences between the 2 intracranial stents according to the analyses of the 2 observers. We believe that this was due to the small sample size of this study. Similarly, previous studies ${ }^{5,21,28}$ have demonstrated that the Neuroform stent generated fewer artifacts than the Enterprise stent because the thinner struts generated less radiofrequency shielding. ${ }^{5,7,11}$

The present study has several limitations. First, the retrospective design is prone to selection bias. Second, there was a long interval between the latest DSA and MRA, which may indicate that the patients were evaluated with MRA when they were at a slightly different status from that in their last DSA. However, we do not regularly perform DSA for patients who do not show evidence of recanalization during follow-up MRA due to the invasiveness of the procedure and the possibility of neurologic complications. Therefore, shorter intervals between the latest DSA and MRA could not be achieved. Additionally, we did not compare our results with those of contrast-enhanced MRA, which is considered a useful follow-up technique for stent-assisted coil embolization. ${ }^{21,29}$ Thus, future studies comparing PETRA qMRA with contrast-enhanced MRA are necessary. Furthermore, we evaluated cases that involved nitinol stents, which have fewer artifacts than stents made using other materials. Further studies should be conducted to determine whether our findings are consistent with the use of stainless steel, platinum, and cobalt stents. Finally, this study had a small sample size; future studies should include a large sample size to confirm the effectiveness of PETRA qMRA.

\section{CONCLUSIONS}

Our results show that PETRA qMRA provides clear visualization of intracranial stent flow in the anterior circulation. Additionally, PETRA qMRA was more similar to the occlusion status of DSA than TOF-MRA. Overall, it appears that PETRA qMRA could be a useful follow-up technique for patients who have undergone stent-assisted coil embolization for anterior circulation aneurysms.

\section{ACKNOWLEDGMENTS}

The authors thank Kang Soo Kim (Siemens Healthcare, Korea) for technical advice.

Disclosures: Young Jin Heo—RELATED: Grant: 2018 Inje University research grant.

\section{REFERENCES}

1. Agid R, Willinsky R, Lee SK, et al. Characterization of aneurysm remnants after endovascular treatment: contrast-enhanced MR angiography versus catheter digital subtraction angiography. AJNR Am J Neuroradiol 2008;29:1570-74 CrossRef Medline

2. Lubicz B, Levivier M, Sadeghi N, et al. Immediate intracranial aneurysm occlusion after embolization with detachable coils: a comparison between MR angiography and intra-arterial digital subtraction angiography. J Neuroradiol 2007;34:190-97 CrossRef Medline

3. Kaufmann TJ, Huston J 3rd, Cloft HJ, et al. A prospective trial of $3 \mathrm{~T}$ and 1.5T time-of-flight and contrast-enhanced MR angiography in the follow-up of coiled intracranial aneurysms. AJNR Am J Neuroradiol 2010;31:912-18 CrossRef Medline

4. Gönner F, Heid O, Remonda L, et al. MR angiography with ultrashort echo time in cerebral aneurysms treated with Guglielmi detachable coils. AJNR Am J Neuroradiol 1998;19:1324-28 Medline

5. Cho WS, Kim SS, Lee SJ, et al. The effectiveness of 3T time-of-flight magnetic resonance angiography for follow-up evaluations after 
the stent-assisted coil embolization of cerebral aneurysms. Acta Radiol 2014;55:604-13 CrossRef Medline

6. Irie R, Suzuki M, Yamamoto $M$, et al. Assessing blood flow in an intracranial stent: a feasibility study of MR angiography using a silent scan after stent-assisted coil embolization for anterior circulation aneurysms. AJNR Am J Neuroradiol 2015;36:967-70 CrossRef Medline

7. Takano N, Suzuki M, Irie R, et al. Usefulness of non-contrast-enhanced MR angiography using a silent scan for follow-up after Yconfiguration stent-assisted coil embolization for basilar tip aneurysms. AJNR Am J Neuroradiol 2017;38:577-81 CrossRef Medline

8. Takano N, Suzuki M, Irie R, et al. Non-contrast-enhanced silent scan MR angiography of intracranial anterior circulation aneurysms treated with a low-profile visualized intraluminal support device. AJNR Am J Neuroradiol 2017;38:1610-16 CrossRef Medline

9. Ye J, Luo X, Qu J, et al. Follow-up assessment of coiled intracranial aneurysms using zTE MRA as compared with TOF MRA: a preliminary image quality study. Eur Radiol 2017;27:4271-80 CrossRef Medline

10. Meyer JM, Buecker A, Spuentrup E, et al. Improved in-stent magnetic resonance angiography with high flip angle excitation. Invest Radiol 2001;36:677-81 CrossRef Medline

11. Choi JW, Roh HG, Moon WJ, et al. Optimization of MR parameters of 3D TOF-MRA for various intracranial stents at 3.0T MRI. Neurointervention 2011;6:71-77 CrossRef Medline

12. Ikushima $Y$, Hashido $T$, Watanabe $Y$, et al. Effects of imaging parameters on the quality of contrast-enhanced MR angiography of cerebral aneurysms treated using stent-assisted coiling: a phantom study. Magn Reson Med Sci 2017;16:146-51 CrossRef Medline

13. Grodzki DM, Jakob PM, Heismann B. Ultrashort echo time imaging using pointwise encoding time reduction with radial acquisition (PETRA). Magn Reson Med 2012;67:510-18 CrossRef Medline

14. Yutaka Natsuaki RG, Grimm R, Grodzki D, et al. PETRA quiet MRA: improved robustness with $3 \mathrm{D}$ elastic cross-registration. In: Proceedings of the Annual Meeting and Exhibition of the International Society for Magnetic Resonance in Medicine, Singapore. May 7-8, 2016

15. Raymond J, Guilbert F, Weill A, et al. Long-term angiographic recurrences after selective endovascular treatment of aneurysms with detachable coils. Stroke 2003;34:1398-403 CrossRef Medline

16. Landis JR, Koch GG. The measurement of observer agreement for categorical data. Biometrics 1977;33:159-74 Medline

17. Santillan A, Greenberg E, Patsalides A, et al. Long-term clinical and angiographic results of Neuroform stent-assisted coil embolization in wide-necked intracranial aneurysms. Neurosurgery 2012;70: 1232-37; discussion 1237 CrossRef Medline

18. Willinsky RA, Taylor SM, terBrugge K, et al. Neurologic complications of cerebral angiography: prospective analysis of 2,899 procedures and review of the literature. Radiology 2003;227:522-28 CrossRef Medline

19. Gauvrit JY, Leclerc X, Pernodet M, et al. Intracranial aneurysms treated with Guglielmi detachable coils: usefulness of 6-month imaging follow-up with contrast-enhanced MR angiography. AJNR Am J Neuroradiol 2005;26:515-21 Medline

20. Gauvrit JY, Leclerc X, Caron S, et al. Intracranial aneurysms treated with Guglielmi detachable coils: imaging follow-up with contrast-enhanced MR angiography. Stroke 2006;37:1033-37 CrossRef Medline

21. Choi JW, Roh HG, Moon WJ, et al. Time-resolved 3D contrastenhanced MRA on 3.0T: a non-invasive follow-up technique after stent-assisted coil embolization of the intracranial aneurysm. Korean J Radiol 2011;12:662-70 CrossRef Medline

22. Schmalbrock P, Yuan C, Chakeres DW, et al. Volume MR angiography: methods to achieve very short echo times. Radiology 1990;175:861-65 CrossRef Medline

23. Robson MD, Gatehouse PD, Bydder M, et al. Magnetic resonance: an introduction to ultrashort TE (UTE) imaging. J Comput Assist Tomogr 2003;27:825-46 CrossRef Medline

24. Fiorella D, Albuquerque FC, Woo H, et al. Neuroform in-stent stenosis: incidence, natural history and treatment strategies. $\mathrm{Neu}$ rosurgery 2006;59:34-42 CrossRef

25. Holdsworth SJ, Macpherson SJ, Yeom KW, et al. Clinical evaluation of Silent T1-weighted MRI and Silent MR angiography of the brain. AJR Am J Roentgenol 2018;210:404-11 CrossRef Medline

26. Bartels LW, Bakker CJ, Viergever MA. Improved lumen visualization in metallic vascular implants by reducing RF artifacts. Magn Reson Med 2002;47:171-80 CrossRef Medline

27. Bartels LW, Smits HF, Bakker CJ, et al. MR imaging of vascular stents: effects of susceptibility, flow, and radiofrequency eddy currents. J Vasc Interv Radiol 2001;12:365-71 CrossRef Medline

28. Lövblad KO, Yilmaz H, Chouiter A, et al. Intracranial aneurysm stenting: follow-up with MR angiography. J Magn Reson Imaging 2006;24:418-22 CrossRef Medline

29. Agid R, Schaaf M, Farb R. CE-MRA for follow-up of aneurysms post stent-assisted coiling. Interv Neuroradiol 2012;18:275-83 CrossRef Medline 